doi:10.13108/2021-13-4-17

\title{
SUM-DIFFERENCE EQUATION FOR ANALYTIC FUNCTIONS GENERATED BY TRIANGLE AND ITS APPLICATIONS
}

\author{
F.N. GARIF'YANOV, E.V. STREZHNEVA
}

\begin{abstract}
Let $D$ be a triangle and $\Gamma$ by the half of its boundary $\partial D$. We consider an element-wise linear sum-difference equation in the class of functions holomorphic outside $\Gamma$ and vanishing at infinity. The solution is sought in the form of a Cauchy-type integral over $\Gamma$ with an unknown density. The boundary values satisfy the Hölder condition on each compact subset in $\Gamma$ containing no nodes. At most logarithmic singularities are admitted at the nodes. In order to regularize the equation to $\partial D$, we introduce a piecewise linear Carleman shift. It maps each side into itself changing the orientation. In this case, the midpoints of the sides are fixed points. We regularize the equation and find its solvability condition for. We consider a particular case when the number of solvability conditions can be counted exactly. We provide applications to interpolation problems for entire functions of exponential type. Previously, similar problems were investigated for tetragon, pentagon, and hexagon.
\end{abstract}

Keywords: sum-difference equation, Carleman problem, equivalent regularization, interpolation problems for entire functions of exponential type.

Mathematics Subject Classification: 30EXX: 30E05, 30E20, 30E25

\section{INTRODUCTION AND FORMULATION OF PROBLEM}

In series of works [1]-[3] there were considered sum-difference equations generated by some polygons. In paper [1] this was a tetragon, while in papers [2] and [3] these were respectively pentagon and hexagon having two equal and parallel sides. Such equations are closely related with interpolation problems for entire functions of exponential type.

Let $D$ be a triangle with vertices at $t_{1}=0, t_{2}=1, t_{3}$, where $\operatorname{Im} t_{3} \neq 0$, and sides $\ell_{j}$ taken in the order of positive bypassing its boundary $\left(t \in \ell_{1} \Rightarrow \operatorname{Im} t=0\right)$. We introduce functions

$$
\sigma_{j}(z)=t_{j}+t_{j+1}-z, \quad j=\overline{1,3}, \quad t_{4}=t_{1} .
$$

These functions map $D$ into triangles having with it a common side. A piece-wise linear function $\alpha(t)=\left\{\sigma_{j}(t), t \in \ell_{j}\right\}$ maps each side $\ell_{j}$ into itself with changing the orientation, and the center of the side $\tau_{j}$ is a fixed point of the shift. Thus, this is an Carleman involutive shift discontinuous at the vertices. The superpositions $\sigma_{j} \sigma_{k}, j \neq k, k=\overline{1,3}$, are of form

$$
\begin{array}{lll}
\sigma_{4}(z)=z-1, & \sigma_{5}=\sigma_{4}^{-1}, & \sigma_{6}(z)=z-t_{3}, \\
\sigma_{7}=\sigma_{6}-1, & \sigma_{8}(z)=z-t_{3}+1, & \sigma_{9}=\sigma_{8}^{-1} .
\end{array}
$$

Extra three transforms

$$
\sigma_{10}(z)=-z, \quad \sigma_{11}(z)=2-z, \quad \sigma_{12}(z)=2 t_{3}-z
$$

F.N. Garif'yanov, E.V. Strezhneva, Sum-Difference Equation for analytic functions generATED BY TRIANGLE AND ITS APPLICATIONS.

(C) Garif'yanov F.N., Strezhneva E.V. 2021.

Submitted November 2, 2020. 
map $D$ into triangles having with it a common vertex. Let $\Gamma$ be a half of the boundary $\partial D$ (a set of segments) satisfying two conditions. First, $\Gamma \cap \alpha(\Gamma)=\varnothing$, and second, $\Gamma \cup \alpha(\Gamma)=\partial D$. These identities are treated up to the vertices in $D$ and the nodes in $\Gamma$. It is clear that $\bar{\Gamma}$ contains at least two vertices and all points $\tau_{j}, j=\overline{1,3}$. First such choice of $\Gamma$ was proposed in paper [4].

We consider a sum-difference equation

$$
(V f)(z) \equiv f(z)+\sum_{m=1}^{12} f\left(\sigma_{m}(z)\right)=g(z), \quad z \in D,
$$

under the following assumptions.

1. A solution $f(z)$ is holomorphic outside $\Gamma$ and vanishes at infinity. Its boundary values $f^{ \pm}(t)$ satisfies Hölder conditions on $\Gamma$. At the nodes $\Gamma$ and the vertices $D$, the solution can have at most logarithmic singularities.

2. A free time $g(z)$ is holomorphic in $D$ and its boundary value satisfies $g^{+}(t) \in H_{\mu}(\partial D)$.

We denote such class of solutions by $B$. A convex envelope of the set $\Gamma$ is a conjugate indicator diagram of an entire function of exponential type $F(z)$ associated in the Borel sense [5, Sect. 1, Subsect. 1] with the lower function $f(z) \in B$. This allows us to apply equation (1.1) to studying interpolation problems for entire functions of exponential type. In Section 2 we propose a method of regularization of equation (1.1). We obtain a condition of equivalence of this regularization. One particular case is studied completely. In Section 3 the obtained results are applied to studying some interpolation problems for entire functions of exponential type.

\section{Regularization of Equation. Particular CASE}

We seek a solution to equation (1.1) as a Cauchy type integral

$$
f(z)=\frac{1}{2 \pi i} \int_{\Gamma}(\tau-z)^{-1} \varphi(\tau) d \tau
$$

with an unknown density $\varphi(\tau) \in H_{\mu}(\bar{\Gamma})$. Then it follows from (1.1) that

$$
(E \varphi)(z) \equiv \frac{1}{2 \pi i} \int_{\Gamma} \varphi(\tau) A(z, \tau) d \tau=g(z), \quad z \in D,
$$

where

$$
A(z, \tau)=(\tau-z)^{-1}+\sum_{m=1}^{12}\left(\tau-\sigma_{m}(z)\right)^{-1} .
$$

Passing to the limit as $z \rightarrow t,(t \in \Gamma)$, by the Sokhotskii formula we obtain

$$
\left(E^{+} \varphi\right)(t) \equiv 2^{-1} \varphi(t)+\frac{1}{2 \pi i} \int_{\Gamma} \varphi(\tau) A(t, \tau) d \tau=g^{+}(t), \quad t \in \Gamma .
$$

Passing to the limit as $z \rightarrow \alpha(t)$, we find

$$
\left(E^{+} \varphi\right) \alpha(t) \equiv-2^{-1} \varphi(t)+\frac{1}{2 \pi i} \int_{\Gamma} \varphi(\tau) A(\alpha(t), \tau) d \tau=g^{+} \alpha(t), \quad t \in \Gamma .
$$


We deduct the second identity from the first one and arrive at the equation

$$
(T \varphi) \equiv \varphi(t)+\frac{1}{2 \pi i} \int_{\Gamma} K(t, \tau) \varphi(\tau) d \tau=g^{+}(t)-g^{+}(\alpha(t))
$$

with the kernel

$$
K(t, \tau)=A(t, \tau)-A(\alpha(t), \tau) .
$$

Lemma 2.1. Kernel (2.4) is bounded.

Proof. The proof is based on straightforward checking the statement for various options of location of the points $\tau$ and $t$ on the sides of the triangle.

Equation (2.3) is a Fredholm equation of second kind. Suppose that it is solvable. Then we go back to equation (1.1). We get:

$$
(2.3) \Rightarrow\left(E^{+} \varphi\right)(t)-\left(E^{+} \varphi\right)(\alpha(t))=g^{+}(t)-g^{+}(\alpha(t)) \Rightarrow(E \varphi)(z)=g(z)+C
$$

since the Carleman problem $a^{+}(t)=a^{+}(\alpha(t))$ possesses a unique solution $a(z)=$ const by a locally conformal gluing principle [6]. The proof is complete.

Theorem 2.1. Problem (1.1) has finitely many, namely, $N+1$ solvability conditions. Among of them, $N$ conditions are the solvability conditions of integral condition (2.3), while one more condition

$$
(E \varphi)\left(z_{0}\right)=g\left(z_{0}\right), \quad z_{0} \in D
$$

ensures the equivalence of the regularization.

We consider a particular case, when we can find the number $N$ exactly. Let $t_{3}=i$ and $\Gamma=\bigcup_{j=1}^{3} \ell_{j}^{\prime}$, where

$$
\ell_{1}^{\prime}=(0,0.5), \quad \ell_{2}^{\prime}=(1,0.5(1+i)), \quad \ell_{3}^{\prime}=(0,0.5 i) .
$$

We consider a homogeneous equation

$$
T \varphi=0 .
$$

We let

$$
M=\max |\varphi(t)|, \quad t \in \Gamma .
$$

We first assume that identity (2.7) is attained as $t \in \ell_{1}^{\prime}$. Under such location of the point $t$ we get:

$$
\begin{aligned}
K(t, \tau)= & (u+i)^{-1}+(u-1-i)^{-1}+(v-2 i)^{-1}-(v-1+i)^{-1} \\
& -(v-2-i)^{-1}-(u+1-2 i)^{-1} \\
= & (2 t-1)\left((u+i)^{-1}(v-1+i)^{-1}+(u-1-i)^{-1}(v-2-i)^{-1}\right. \\
& \left.-(v-2 i)^{-1}(u+1-2 i)^{-1}\right)
\end{aligned}
$$

where $u=\tau-t, v=\tau+t$.

The absolute value of the kernel attains its maximal value at $t=0$, while the absolute value of each term in square brackets does not exceed 1 and taking into consideration the lengths of the segments $\ell_{j}^{\prime}$ we obtain:

$$
3\left(1+\frac{1}{\sqrt{2}}\right)<2 \pi
$$

which implies $\varphi \equiv 0$. 
Suppose that identity (2.7) is attained as $t \in \ell_{2}^{\prime}$. Then

$$
\begin{aligned}
K(t, \tau) & =(u-1)^{-1}+(u-i)^{-1}+v^{-1}-(v+2-i)^{-1}-(v-1-2 i)^{-1}-(u+1+i)^{-1} \\
& =(2 t-1-i)\left((u-1)^{-1}(v-2-i)^{-1}+(u-i)^{-1}(v-1-2 i)^{-1}-v^{-1}(u+1+i)^{-1}\right) .
\end{aligned}
$$

The maximum of the absolute value of the kernel is attained at $t=1$ and the absolute value of the sum in the square brackets does not exceed 2.17, that is,

$$
\left(1+\frac{1}{\sqrt{2}}\right)|K(t, \tau)| \leq 6,
$$

and this again implies that $\varphi \equiv 0$.

It remains to suppose that identity $(2.7)$ is attained at $t \in \ell_{3}^{\prime}$. Then

$$
\begin{aligned}
K(t, \tau)= & (u+1)^{-1}+(u-i+1)^{-1}+(u-2)^{-1}-(v-i+1)^{-1} \\
& -(v+1-2 i)^{-1}-(u-2+i)^{-1} \\
= & (2 t-1)\left((u+1)^{-1}(v-i+1)^{-1}+(u-i+1)^{-1}(v+1-2 i)^{-1}\right. \\
& \left.-(v-2 i)^{-1}(u-2+i)^{-1}\right) .
\end{aligned}
$$

The absolute value of the kernel attains its maximum at $t=0$, while the absolute value of each term in square brackets does not exceed 1 and taking into consideration the lengths of the segments $\ell_{j}^{\prime}$ we obtain

$$
3\left(1+\frac{1}{\sqrt{2}}\right)<2 \pi
$$

and this again implies that $\varphi \equiv 0$. We note that in this case we do not need sharper estimates. Thus, homogeneous equation (2.6) possesses only the trivial solution.

Theorem 2.2. In the considered example $N=0$, that is, problem (1.1) possesses one solvability condition (2.5).

\section{APPLiCATIONS}

Equation (1.1) is closely related with interpolation problems for entire functions of exponential type. We restrict ourselves by considering a following example. Let $F(z)$ be an entire function of exponential type associated in the Borel sense with the lower function $f(z) \in B$. Its indicator diagram is a trapezoid $D_{0}$ with vertices at $0,1,(i+1) / 2, i / 2$.

Remark 3.1. A conjugate indicator diagram can be a smaller convex set $D^{\prime} \subset D_{0}$. In order this to be the case, it is necessary but not sufficient the free term $g(z)$ to be analytically continued from $D$ into some neighbourhood of the infinity and $g(\infty)=0$. Then relation (1.1) holds not only as $z \in D$, but also in this neighbourhood, that is, the problem is overdetermined and its solution can be obtained in an explicit form. Such case is not of a great interest; for more details see [2].

We take a triangle $\Delta_{1}=D \backslash \overline{D_{0}}$ and let $z_{0} \in \Delta_{1}$. We also let

$$
g(z)=C_{0}+\sum_{k=1}^{\infty} \frac{C_{k}\left(z-z_{0}\right)^{k}}{k !}
$$

and suppose that the convergence radius of this series is $R>\left|z_{0}-1\right|$. 
For $z \in \Delta$ we rewrite relation (1.1) in the form

$$
H(z) \equiv \int_{\theta_{0}} F(\tau) \exp (-z \tau) d \tau+\sum_{k=1}^{12} \int_{\theta_{k}} F(\tau) \exp \left(-\tau \sigma_{k}(z)\right) d \tau=g(z), \quad z \in \Delta .
$$

Here $\theta_{k}$ is the ray $\arg \tau=-\pi / 2$ for $k=0,4,5,7,9,12$, the ray $\arg \tau=\pi / 2$ for $k=1,6,8,10,11$, the ray $\arg \tau=-\pi / 4$ for $k=2$ and the ray $\arg \tau=\pi$ for $k=3$. The coefficient $C_{0}$ is chosen so that solvability condition (2.5) holds. Equating the Taylor coefficients in the left and right hand sides of the identity at the point $z_{0}$, we obtain

$$
\left.\frac{d^{k} H(z)}{d z^{k}}\right|_{z=z_{0}}=C_{k}, \quad k=\overline{1, \infty} .
$$

Theorem 3.1. Interpolation problem (3.2) for an entire function of exponential type $F(z)$ associated in the Borel sense with the lower function $f(z) \in B$ is solvable and has a unique solution.

Now we return back to the general case and make some remarks.

Remark 3.2. Let $t_{j} \in \bar{\Gamma}$ for all $j$, that $i s, D_{0}=D$. Then there arise non-classical interpolation problems for entire functions of exponential type. A general impression on such problems is given by work [7]. Here the authors considered a problem on recovering an entire function of exponential type by identities relating the Taylor coefficients of the lower function with the Stieltjes momenta of the upper function with respect to some exponential weight.

Remark 3.3. As it has been already mentioned, there is some freedom in choosing the set $\Gamma$, hence, also in the choice of the conjugate indicator diagram $D_{0}$. In particular, for all $\gamma \in[0.75,1]$ one can choose $\Gamma$ so that the quotient of the area $D_{0}$ to the area of the triangle is equal to $\gamma$.

Remark 3.4. Let the closure $\bar{\Gamma}$ contains only two vertices. Then the proposed regularization method can be also applied to the equations obtained by excluding some terms from the operator $V$. The matter is that some terms in (2.2) are bounded as $z=t \in \Gamma$. This is why in (1.1) we can omit the terms corresponding to such $\sigma_{m}$. For instance, in the considered example we can choose all or some of five terms corresponding to the transforms $\sigma_{m}, m=\overline{6,9}$, and $\sigma_{12}$. But the transforms $\sigma_{m}, m=\overline{0,3}$, can not be omitted for any choice of $\Gamma$. Moreover, let $b(z) \in A[D]$ and $b(\bar{D}) \cap \bar{D}=\varnothing$. Then we can regularize the equation

$$
(V f)(z)+f(b(z))=g(z), \quad z \in D .
$$

Let us compare these results with ones obtained earlier for other $n$-gons. We denote by $p$ the minimal number of the terms in the left hand side of the corresponding sum-difference equation, under which it admits the regularization in the above way. For the sake of definiteness we suppose that the set $\bar{\Gamma}$ contains all vertices of the polygon, see Remark 3.4. Then for $n=4$ we have $p=9$ [1], for $n=5$ we have $p=7$ [2] and for $n=6$ we have $p=7$ [3]. But for $n=3$ we get $p=13$.

\section{BIBLIOGRAPHY}

1. F.N. Garif'yanov. Multielement equations for analytic functions in the plane with cuts // Sibir. Matem. Zhurn. 57:2, 276-281 (2016). [Siberian Math. J. 57:2, 213-217 (2016).]

2. E.P. Aksenteva, F.N. Garifyanov. Sum-difference equation for analytic functions generated by pentagon and its application // Lobachevskii J. Math. 37:2, 101-104 (2016). 
3. F.N. Garif'yanov, E.V. Strezhneva. Sum-difference equation for analytic functions, generated by a hexagon, and its applications // Izv. VUZov. Matem. 7, 56-62 (2020). [Russ. Math. (Iz. VUZ). 64:7, 48-53 (2020).]

4. F.N. Garif'yanov, S.A. Modina. The Carleman kernel and its applications // Sibir. Matem. Zhurn. 53:6, 1263-1273 (2012). [Siberian Math. J. 53:6, 1011-1020 (2012).]

5. L. Bieberbach. Analytische Fortsetzung. Springer-Verlag, Berlin (1955). [Analytic continuation. Moscow, Nauka (1967). (in Russian).]

6. Eh. I. Zverovich. A method of locally conformal gluing // Dokl. AN SSSR. 205:4, 767-770 (1972). [Sov. Math. Dokl. 13, 1003-1007 (1972).]

7. F.N. Garifyanov, D.B. Kats. On an equation with Carleman kernel and its application to the moment problem // Uchenye Zapiski Kazan. Univ. Ser. Fiz.-Matem. Nauki. 154:3, 112-120 (2012). (in Russian).

Farkhat Nurgayazovich Garif'yanov

Kazan State Power Engineering University

Krasnosel'skaya str. 51

420066, Kazan, Russia

E-mail: f.garifyanov@mail.ru

Elena Vasilievna Strezhneva,

Kazan National Research Technical

University named after A. N. Tupolev - KAI

Karl Marx str. 10

420111, Kazan, Russia

E-mail: strezh@yandex.ru 